\title{
Philonsorbonne
}

\section{Langage et pouvoir dans le Traité politique de Spinoza}

\section{Céline HERVET}

\section{(2) OpenEdition \\ Journals}

Édition électronique

URL : https://journals.openedition.org/philonsorbonne/109

DOI : 10.4000/philonsorbonne.109

ISSN : 2270-7336

Éditeur

Publications de la Sorbonne

\section{Édition imprimée}

Date de publication : 15 novembre 2007

Pagination : 49-63

ISBN : 978-2-85944-594-2

ISSN : $1255-183 X$

\section{Référence électronique}

Céline HERVET, « Langage et pouvoir dans le Traité politique de Spinoza », Philonsorbonne [En ligne], 1 | 2007, mis en ligne le 27 janvier 2013, consulté le 08 juin 2021. URL : http://journals.openedition.org/ philonsorbonne/109; DOI : https://doi.org/10.4000/philonsorbonne.109 


\title{
Langage et pouvoir dans le Traité politique de Spinoza
}

\author{
Céline Hervet
}

Quel est le véritable pouvoir dévolu à la parole dans le champ politique ? Le langage y produit-il des effets ? Si le domaine des affaires publiques doit son existence aux discours des uns et des autres, aux ordres et aux commandements du pouvoir souverain, aux discussions qui précèdent la prise d'une décision, aux promesses qui permettent de briguer ou de conserver les faveurs de la multitude, aux rumeurs et aux déclarations qui agitent le corps politique, ce n'est pas, semble-t-il, en vertu d'une puissance (potentia) inhérente au langage considéré en lui-même. L'ordre comme commandement n'est pas fondateur ni instaurateur d'un ordre comme organisation politique. À la différence de Hobbes, il ne suffit pas pour Spinoza de dire, d'édicter, pour faire exister et faire respecter le pouvoir souverain. Le langage n'a pas en lui-même cette faculté de créer un ordre politique, comme pourrait le laisser penser la fondation mosaïque de l'État hébreu décrite dans le Traité théologico-politique. Or cette relative impuissance de la parole, qui ne peut à elle seule soutenir l'existence d'un régime, trouve son origine dans une réflexion sur le langage qui traverse le corpus spinoziste. Ces remarques sur le langage qui parsèment tous les textes de Spinoza me semblent expliquer sa réflexion assez pragmatique sur les théories du contrat social, celui-ci ne pouvant prendre la forme d'un engagement originel qui contraindrait les hommes pour l'avenir. Cette parole originelle ne peut à elle seule fonder un ordre futur. Comment expliquer cependant à la fois l'insistance de Spinoza à vider toute parole, toute promesse, tout engagement de son contenu au motif que les mots ne sont rien face à la puissance et n'ont aucune effectivité au sein de rapports de force, et dans le même temps, car ceci a lieu précisément dans le Traité politique, sa défense, son plaidoyer pour les processus de délibérations, de discussion dans le cadre institutionnel imaginé pour les régimes tant monarchique qu'aristocratique. La question de la démocratie et de son organisation institutionnelle demeurant ouverte. Les mots sont impuissants, 
mais tout régime politique doit laisser les dissensions, les conflits trouver leur expression, une expression verbale.

\section{Éléments de la philosophie du langage de Spinoza}

Cette philosophie du langage peut se résumer en trois points principaux : premièrement, le langage n'a pas de rapport immédiat à la réalité, il n'a en lui-même aucune teneur ontologique, si bien que pour parler des mots, Spinoza quitte tout à fait le discours de la référence et de l'opposition vrai/faux. Deuxièmement, les mots se rattachent à une connaissance imparfaite, celle du premier genre, et ne sont pas le signe d'une vérité et d'une rationalité sans faille, puisqu'ils sont liés dans leur constitution même à l'imagination. Troisièmement, il n'y a pas de langage concevable en dehors des usages historiques, des situations d'énonciation qui obéissent au critère de l'utilité. Ces trois caractéristiques du langage permettent de redéfinir ce qu'il faut entendre par le sens d'un mot.

\section{Inadéquation à l'égard de la réalité}

Commençons par cette critique des mots au motif de leur inadéquation à l'égard du réel qu'ils prétendent signifier. Elle est présente dès les Pensées métaphysiques qui nous font pénétrer de plain-pied dès le premier chapitre dans le nominalisme de Spinoza. Dans ce chapitre, intitulé «De l'Être réel, de l'Être Forgé et de l'Être de raison ", Spinoza s'attache à circonscrire la sphère du réel, en excluant de celle-ci ce que la philosophie scolastique appelait «être de raison». C'est autour de ces êtres de raison, définis comme des modes de penser qu'utilise l'esprit afin de se rapporter au réel selon ses propres règles, servant «à retenir, expliquer et imaginer plus facilement des choses connues $»^{1}$ que se cristallise le risque de confusion entre l'être et le non-être ( «Il n'y a aucune concordance entre l'Être réel et les objets auxquels se rapporte l'Être de raison $\left.»^{2}\right)$. Les mots apparaissent comme des aide-mémoire qui indiquent une réalité en en résumant pour les besoins de l'esprit les principaux aspects : c'est parce qu'il n'a aucune réalité ontologique que le langage possède une fonction, une utilité dans la démarche de connaissance. Le signe verbal possède une extension inversement proportionnelle à sa réalité ontologique.

C'est bien du point de vue d'une séparation nette entre le mot et la chose que Spinoza aborde la nature du nom. L'accord strictement nominal entre un signe ou un aide-mémoire et la chose qu'il désigne et qu'il est censé rappeler est le plus bas degré de convenance qui puisse exister : "pour retenir une chose tout à fait nouvelle et l'imprimer dans la mémoire, nous avons recours

1. Pensées métaphysiques, trad. Appuhn, éd. G.-F., p. 337.

2. Ibid., p. 340 . 
à une autre chose qui nous est familière et qui s'accorde avec la première soit seulement par le nom, soit en réalité $»^{3}$. Le nom se situe donc en dehors de ce que Spinoza appelle la réalité, et se rattache à la catégorie des «modes de penser » qui traitent la réalité, en font une matière connaissable que l'intellect peut appréhender. C'est de l'être réel et non des mots qu'il faut partir, les mots n'étant que les instruments de l'intellect : seul l'intellect en tant qu'il est actif possède une réalité à l'égard de laquelle la nature des mots est d'ordre fonctionnel. Les mots, lorsqu'ils sont assemblés au sein d'une définition, obéissent à l'usage stratégique qui détermine l'utilisation de telle définition à tel moment de l'argumentation.

Il n'est pas plus faux de parler de l'homme comme d'un « animal bipède sans plumes », que de le définir comme un «animal raisonnable», puisque la valeur de cette définition est exclusivement fonctionnelle. Le langage est conçu comme un instrument, un outil de connaissance. Dégagé de l'exigence de référence et d'adéquation à la réalité, les mots constituent du même coup un lieu d'invention où tout semble pouvoir se dire. C'est ce que montrent les Pensées métaphysiques au chapitre III à travers la définition de la chimère que Spinoza associe étroitement à celle du langage : « une chimère, n'étant ni dans l'entendement ni dans l'imagination, peut être appelée proprement par nous un être verbal ; car on ne peut l'exprimer autrement que par des mots. Par exemple nous exprimons par le langage un cercle carré, mais nous ne pouvons l'imaginer en aucune façon et encore bien moins le connaître. C'est pourquoi une chimère n'est rien qu'un $\operatorname{mot}^{4}$. » Or ce que l'on doit retenir ici et qui va nous intéresser par la suite, c'est que, ce que l'on ne peut que dire n'existe pas, ce qui n'existe que dans les mots n'existe pas. Et les mots sont alors le moyen de déraisonner, de quitter le domaine des choses réelles.

\section{Origine imaginative du langage}

On en arrive alors au deuxième point, c'est-à-dire l'absence de rationalité intrinsèque du langage. Du point de vue de la connaissance, le langage ne peut conduire qu'à une connaissance du premier genre. Le Traité de la réforme de l'entendement signale bien le lien entre la nomination, entre la fabrication des noms et l'imagination. Le mot n'est pas la traduction dans l'élément verbal de l'idée, mais ce qui correspond dans le langage à une image mentale, abstraction fictive d'une pluralité d'éléments singuliers. Dans le Court Traité, l'Éthique, et dans le Traité de la réforme de l'entendement, la connaissance du premier genre, par ouï-dire, est clairement définie comme verbale et ne fait reposer la certitude que sur les mots d'autrui, qui ne peuvent suppléer son manque de fondement rationnel. Citons simplement le Traité de la réforme de l'entendement, § 19: «Il y a la perception que nous avons à partir du ouï-dire ou de quelque signe, qu'on

\footnotetext{
3. Op. cit., p. 338.

4. Op. cit., p. 345-346.
} 
appelle arbitraire ». Si les mots ne se réfèrent à la réalité que de manière imparfaite voire trompeuse, c'est donc à cause de leur lien originaire avec l'imagination. À ce titre, le Traité de la réforme de l'entendement nous fournit le texte le plus explicite : «les mots ont été constitués au gré et à la portée des gens ordinaires, en sorte qu'ils ne sont que des signes des choses, conformes à ce qu'elles sont dans l'imagination et non à ce qu'elles sont dans l'intellect : ce qui ressort clairement de ceci, qu'à toutes celles qui sont seulement dans l'intellect et non dans l'imagination, ils ont souvent donné des noms négatifs, comme sont "incorporel”, "infini”, etc. » Un nom doit renvoyer aisément à sa propre trace dans l'imagination, si bien que son aspect matériel dépend du fonctionnement de l'imagination plutôt que de l'intellect. On comprend déjà à travers cette solidarité ce lien étroit entre le langage et l'imagination, ce qui peut inquiéter un pouvoir souverain qui tenterait d'imposer à la multitude son propre discours sous prétexte qu'il serait celui de la raison, en interdisant l'expression de paroles et d'opinions concurrentes, qui n'obéiraient pas aux mêmes règles.

On voit aussi dans ce lien posé entre langage et imagination la faculté inhérente au discours de quitter l'ordre du réel. En effet, cette définition fonctionnelle du langage et cette solidarité entretenue avec l'activité imaginative le situe en marge des critères de la vérité et de l'erreur. Les mots peuvent en effet tout dire : il y a d'un côté ce que je sais, ce que je sais être vrai et de l'autre ce que je dis. Or le langage ne m'oblige pas à l'égard de la vérité, il constitue un espace où se meuvent, selon mon choix, selon ma fantaisie, le vrai comme le faux. Le Traité de la réforme de l'entendement présente de nombreux exemples de cette dissociation entre les possibilités qu'offre le langage, les connexions et les associations qu'il permet, et celles de la pensée : l'élément verbal apparaît à de nombreuses reprises comme le lieu de la fabulation, voire de l'expérimentation intellectuelle. Là où l'intellect ne peut pas aller, le langage lui, peut dire, affirmer de manière presque illimitée. Cette plasticité du langage, capable de servir toutes les fantaisies possibles, est d'autant plus importante que notre connaissance des choses est faible, incertaine et permet de ce fait les expériences lexicales les plus inattendues, telles les contradictions dans les termes.

Or c'est cette déconnexion toujours possible entre langage et vérité qui est à l'origine de toutes les affabulations, de tous les mensonges. Les chimères comme les manipulations les plus perverses sont permises par le langage : je peux dire que «la terre est un demi-globe et comme une demiorange dans une soucoupe ou que le soleil se meut autour de la terre », ou qu'Alexandre est fils de dieu, même si je sais que cela est faux, il se peut que celui à qui je le dis me croit et reste dans une ignorance à laquelle les mots ont pu contribuer.

\section{Matérialisme de la langue}

Le langage est entièrement le produit d'une histoire, qui définit et redéfinit, au gré des changements politiques et sociaux, le sens des mots. Ce 
point de vue résolument matérialiste sur le langage est perceptible dès les Pensées métaphysiques au chapitre VI de la première partie. Dans ce chapitre, Spinoza se livre à une histoire des significations des termes «vrai » et «faux», et fort de ses découvertes, analyse les termes «bon» et « mauvais ». Or il apparaît que ces termes sont tous relatifs à un état social et qu'il faut y voir le résultat d'une histoire et des besoins à un moment précis des locuteurs. Ainsi, ce fut d'abord pour qualifier les récits qu'on a employé les termes de vrai ou de faux, et ce n'est que par une succession de métaphores que nous en sommes arrivés à attribuer ces termes à des idées et des choses inertes, comme si celles-ci racontaient des histoires sur ellesmêmes. C'est en oubliant cette origine matérielle, ce besoin de signifier qui font des mots des auxiliaires utiles que les philosophes ont fini par tomber dans les pièges du langage ${ }^{5}$.

Cette définition matérialiste du langage est reprise avec plus de force dans le Traité théologico-politique lorsqu'il est question au chapitre XII ( «La parole de Dieu »), § 5, des mots «sacré » et «divin»: «On appelle sacré et divin ce qui est destiné à la pratique de la piété et de la religion ; cela sera sacré aussi longtemps que les hommes s'en serviront religieusement ». Le sens des mots est tributaire de conditions matérielles concrètes. Ce matérialisme est une conséquence logique de la philosophie immanentiste qu'est celle de Spinoza : rien n'est réel que ce qui existe effectivement, les mots n'ont donc de réalité et de sens que s'ils se réfèrent à quelque chose d'existant, c'est-à-dire à une pratique, à un usage. Cela permet à Spinoza de redéfinir la valeur de tout texte sacré : «c'est du seul usage que les mots tirent une signification déterminée ; si, conformément à leur usage, ils sont disposés de sorte que ceux qui les lisent soient portés à la dévotion, alors ces mots seront sacrés ainsi que le livre écrit avec un tel arrangement de termes. Mais si cet usage disparaît ensuite, de sorte que ces mots n'aient plus de signification, ou bien si, soit par méchanceté, soit qu'on n'en ait plus besoin, le livre est complètement négligé, alors les mots comme le livre n'auront plus ni usage ni sainteté. » Ainsi la portée des paroles, qu'elles soient d'ordre religieux ou politique, n'est jamais inscrite éternellement dans la pierre, par conséquent ce n'est pas le langage qui a le pouvoir de faire obéir le croyant, mais l'usage que celui-ci en fait, correspondant à des conditions extérieures au langage lui-même. Le pouvoir de contrainte d'un mot, d'un discours est donc à chercher ailleurs que dans les mots eux-mêmes.

Ces quelques remarques sur la conception spinoziste du langage offrent une définition originale de la notion de sens : un mot ne doit pas son sens à son adéquation à la réalité, puisque les mots ne se rapportent véritablement qu'au travail de l'imagination ; on a vu également que le sens obéit à une intention de signifier, à la fonction que le locuteur confère aux mots qu'il emploie au sein du discours qu'il ordonne ; enfin le sens évolue avec l'usage

5. J'emprunte cette expression aux analyses nietzschéennes de l' «Introduction théorétique sur la vérité et le mensonge au sens extra moral», dans Le Livre du philosophe, p. 123 notamment, éd. G.-F., trad. Angèle Kremer-Marietti. 
dont il est entièrement tributaire. C'est pourquoi il n'y a pas chez Spinoza cet idéal classique d'une langue parfaite, entièrement formelle, conçue en dehors d'un usage réel, matériel et historique des mots. Dès lors on peut se demander quelle est la valeur du langage dans le champ politique. Comment s'y prend-on pour contraindre si ce n'est par les mots, les ordres, les commandements?

\section{Y a-t-il un pouvoir du langage ?}

Pour répondre à cette question, il faut suivre la logique des développements spinozistes qui, au moment même où ils s'attachent à élaborer les fondements de l'ordre politique, et à discuter l'idée d'un contrat originel, passe par une analyse de la promesse. On l'a vu, aucune valeur ontologique n'est conférée au langage, mais c'est une conception exclusivement fonctionnaliste qui se dévoile dans les textes spinozistes sur le langage. Les mots servent à dire et à agir, et ne révèlent en eux-mêmes aucune vérité. Ils servent à mentir, à influencer, comme à accomplir toutes ces procédures utiles à la recherche de la vérité, à la démarche de connaissance. L'essentiel est qu'ils fonctionnent, jouent leur rôle d'outils de l'exercice de la puissance et s'ils ne fonctionnent plus, s'ils s'avèrent trop inadéquats à la réalité qui se joue indépendamment d'eux, alors il ne faut en aucun cas les conserver comme tels et leur conférer un sens, c'est-à-dire un pouvoir qu'ils n'ont plus. Le cas de la promesse constitue un modèle particulièrement fructueux, car celle-ci consiste précisément dans un engagement pour l'avenir que les mots sont censés contenir; lorsque je promets, je m'engage à donner aux mots que j'utilise le même sens aujourd'hui et demain. On comprend alors que la promesse n'ait de valeur selon Spinoza que conjoncturelle, compte tenu d'un certain état des forces en présence, et que je puisse rompre mon engagement en toute bonne foi, puisque si je n'ai plus la puissance et donc la liberté de faire qu'aujourd'hui soit comme hier, c'est-à-dire de maintenir en l'état le rapport de force, mes paroles, sont nulles et non avenues. Si celui qui a promis par exemple d'obéir à un autre qui le surpassait en termes de puissance voit le rapport s'inverser, il n'est plus tenu d'obéir, car comme le dit Spinoza au chapitre II, $\S 12$, du Traité politique, «il n'a donné que des mots ».

Or, l'institution du corps politique passe nécessairement, que ce soit de manière effective ou seulement symbolique, par une parole conçue comme acte fondateur. Le droit est avant tout ce qui est dit, exposé à l'attention de ceux qui, au moment même où ils reçoivent cette parole, changent de statut en devenant sujets d'un pouvoir (potestas). Le discours politique doit donc bien exercer une action sur ses auditeurs, et provoquer des effets moraux susceptibles de fonder durablement l'existence de la société politique. Or c'est un tout autre processus que l'on observe chez Spinoza, et dont le Traité théologico-politique comme le Traité politique offrent la description précise 
et surprenante. $\mathrm{Au}$ commencement de la vie sociale, nul «Verbe» instaurateur d'un ordre en rupture avec ce qui l'a précédé, mais un effort, une puissance. Le fonctionnement réel qui sous-tend le pacte social, la décision de mener une vie commune prennent appui sur cette rationalité instrumentale la plus élémentaire fondée sur l'exigence naturelle de l'autoconservation. Le langage apparaît alors soumis à la recherche individuelle de l'utile, et se trouve renvoyé non pas au rôle instaurateur d'un ordre politique artificiel, mais à celui d'instrument du conatus. Les paroles ne sont qu'un moyen parmi d'autres pour convoiter ce qui nous permet d'acquérir davantage de puissance, ou de persévérer dans notre être. Le langage ainsi instrumentalisé n'exprime que la naturalité du désir auquel s'ordonnent toutes nos actions. Même les paroles les plus sensées, même les prières les plus pieuses trouvent leur origine dans cet appétit qui pousse les sages à être sages, et les déments à se tenir en dehors de la raison. Citons à ce propos le chapitre XVI, $\S 2$ du Traité théologico-politique: «Ce que chacun, considéré sous le seul empire de la nature, juge utile pour lui-même, que ce soit sous la conduite de la saine raison ou sous l'impulsion des affects, il lui est permis, par un souverain droit de nature, de le convoiter et de le saisir de n'importe quelle manière, par force, par ruse, par des prières ou de toute autre façon qui lui sera la plus commode. » Le langage de la loi se place lui aussi du côté de l'utile : les prescriptions ne sont pas bonnes en elles-mêmes, mais constituent un discours adaptée à une vie en commun qui répond aux nécessités dictées par l'instinct, le besoin de sécurité.

Spinoza est ici très proche de Machiavel qui affirme que nul n'est contraint de respecter une promesse faite sous la contrainte de la force ${ }^{6}$. Celui qui rompt une telle promesse n'est pas déshonoré puisqu'il ne fait qu'exercer son droit. Seule la menace immédiate a force de droit, cette force disparaissant avec elle. Une promesse est inséparable des conditions spécifiques de son énonciation qui ne lui confèrent jamais qu'une validité limitée. Or si l'on applique cet argument à l'ordre politique dont il est question dans les chapitres XVI à XX du Traité théologico-politique, ainsi qu'aux chapitres II et III du Traité politique, on s'aperçoit combien celui-ci est précaire s'il ne repose que sur un tel énoncé. Il faudrait en effet reproduire à chaque instant cette peur du danger qui conduit les individus à renoncer au droit naturel qu'ils ont sur toutes choses (c'est ce qu'affirme Hobbes au chapitre XIV du Léviathan : la peur et l'orgueil sont les ressorts de l'obéissance que recèle la nature humaine).

La valeur et le sens de la promesse sont donc directement liés aux effets pratiques d'une telle énonciation, et l'on peut qualifier de pragmatique ici l'interprétation que propose Spinoza de la promesse et plus généralement de toute fondation verbale et juridique de la souveraineté si l'on admet qu'une des définitions possibles de la pragmatique consiste dans «l'étude de tout ce qui, dans le sens d'un énoncé, tient à la situation dans laquelle l'énoncé est 
émis ou employé » ${ }^{7}$. La signification et l'effet pratique d'une promesse sont issus d'une situation de discours, la menace pressante de la violence anarchique des rapports humains qui forme le contexte matériel sans lequel elle n'est qu'une parole creuse. C'est la connaissance de l'enchaînement des événements précédents l'acte de promettre qui peut restituer son intelligibilité à cet engagement qui sans cela paraît contre-nature, la promesse résultant d'un processus au sein duquel la parole trouve sa nécessité et son effet. De même la relation qui existe entre les deux interlocuteurs est à prendre en compte pour qui veut comprendre le sens, c'est-à-dire la validité, de la promesse : promettre quelque chose, «le couteau sous la gorge » ne peut être mis sur le même plan qu'une promesse faite, de mon plein gré, du moins à ce moment-là, à un ami. Or la promesse est réduite par Spinoza à cette situation précise où les individus doivent se défendre contre un mal qui ferait obstacle à leur puissance, autrement dit, la situation de discours qui est celle de toute promesse et ce, en vertu de la nature humaine qu'il décrit, est la perspective de la souffrance, de la violence, voire de la mort.

La thèse fondamentalement spinoziste d'un développement spontané des forces, d'une production immédiate qui traverse sa métaphysique comme sa politique et qui a pu être définie comme une «ontologie de la puissance » rend inutile l'appel à une médiation telle que le contrat social, ainsi qu'il se trouve décrit chez Hobbes. Spinoza explique la différence fondamentale qui existe entre sa philosophie et celle de Hobbes dans la Lettre 50 à Jarig Jelles $^{8}$ par le fait que le contrat ne consiste jamais dans une rupture avec l'état de nature : derrière l'engagement des hommes à vivre ensemble et à confier le pouvoir à l'un d'entre, il faut toujours voir le discours de la puissance. Le pouvoir du chef politique est relatif au degré de puissance qu'il est capable d'exercer sur ses concitoyens. Cette ontologie de la puissance a pour conséquence un anti-juridisme, un rejet de la médiation juridique d'un pouvoir souverain au profit de la puissance de la multitude et des forces sociales ${ }^{9}$.

Chez Hobbes, en effet, le contrat ne peut subsister sans que des signes en soient donnés: "Le moyen par lequel on renonce ou transfère simplement son droit est une déclaration ou une signification à l'aide de signes volontaires et suffisants par lesquels on renonce ou transfère effectivement [...] ce droit à celui qui l'accepte. Ces signes sont ou bien des mots seulement, ou bien des actions seulement : ou (comme c'est le plus

7. O. Ducrot et J.-M. Schaeffer, Nouveau dictionnaire encyclopédique des sciences du langage, Seuil, coll. « Points », p. 131.

8. «Vous me demandez quelle différence il y a entre Hobbes et moi quant à la politique : cette différence consiste en ce que je maintiens toujours le droit naturel et que je n'accorde dans une cité quelconque de droit au souverain sur les sujets que dans la mesure où par la puissance, il l'emporte sur eux : c'est la continuation de l'état de nature », trad. Ch. Appuhn, Gallimard-Flammarion., p. 83.

9. Voir les analyses de G. Deleuze dans sa préface à L'Anomalie sauvage d'Antonio Negri. 
souvent le cas) à la fois des mots et des actions $»^{10}$. Le transfert de droits et l'engagement qui définit le contrat a besoin d'un signe exprès qui en soit la matérialisation: c'est le rôle conféré à la promesse, seule garantie de l'application et du respect du pacte par les contractants. On voit donc que le fondement du contrat est essentiellement verbal, et qu'il repose sur une théorie spécifique de l'autorité et du poids de la parole dans le champ politique et a fortiori de la parole donnée. Hobbes ne cesse de louer l'invention de la parole conçue comme un instrument éminemment politique, puisqu'elle constitue une première forme d'échange entre des individus que l'état de nature tenait isolés les uns des autres (Léviathan I, 4). En utilisant le langage, en signifiant le monde extérieur, l'homme accède à une dimension d'altérité nécessaire à l'effectuation du pacte. Le langage apparaît comme un instrument de socialisation, et peut même être conçu comme l'élément dans lequel les hommes se confrontent les uns aux autres et établissent des structures d'échanges. Il faut se rappeler que la définition hobbesienne du contrat vaut pour tout type de convention, et pas seulement celle spécifique du contrat ou du pacte social, le langage intervenant à titre essentiel, comme condition matérielle de l'engagement mutuel, qui s'extériorise ainsi dans un discours intentionnel venant lier les deux contractants par une parole donnée et reçue. Le langage est bien le milieu «naturel» de la sociabilité, et les serments sont une monnaie d'échange nécessaire à la continuité de la vie sociale. Seules les paroles échangées devant témoins peuvent servir de référent objectif à un engagement qui sans elles resterait illusoire, et ne pourrait fonder la confiance mutuelle exigée par la vie en société.

Malgré tout, si la parole est privée de ce pouvoir impératif, du moins au moment inaugural que constitue le pacte social, il semble en revanche que le pouvoir politique, dans son exercice même, ne puisse pas faire l'économie d'une action sur le langage, à défaut d'une action par le langage et la parole instauratrice. Par deux fois dans le Traité théologico-politique, Spinoza cite cette maxime de Quinte-Curce : "On ne commande pas aux âmes comme on commande aux langues », chaque fois en la nuançant sous une forme concessive, pour en diminuer la portée. Il semble possible à celui qui gouverne d'agir non seulement sur les langues, c'est-à-dire de maîtriser le « débat public », de faire le partage entre ce qui peut se dire et ce qui ne le peut pas, mais aussi, et c'est là le point important que Spinoza met en lumière, sur les esprits. Ce pouvoir sur ses sujets que le prince peut avoir s'appuie sur la mise en œuvre de moyens indirects, diffus, mais qui n'en sont pas moins efficaces. La séparation que posait Quinte-Curce, et que reprendra Hobbes, entre ce qui relève d'un for interne, nos croyances propres, notre intime conviction, et ce qui relève d'un for externe, nos actes extérieurs, qui peuvent nous être reprochés, ainsi que nos paroles, est supprimée par Spinoza, pour qui les esprits sont aussi accessibles au souverain que les paroles énoncées. Aucune barrière n'est plus infranchissable pour le pouvoir, s'il sait s'y prendre. Le seul espace de liberté dévolue à l'intériorité, qui

10. Léviathan, I, chapitre XIV, Gallimard-Folio, p. 233-234. 
subsistait encore dans des théories politiques de type absolutiste disparaît, ouvrant la voie à un gouvernement des esprits. Il est possible en effet de lire cet effacement de l'impénétrabilité de l'âme humaine comme l'ébauche d'une théorie de la propagande politique, dont l'enjeu serait, au moyen de discours concertés, de faire croire à autrui ce qui intéresse le pouvoir. La parole politique qui se mue en tromperie délibérée, provoque des effets en s'adressant non plus à l'esprit, mens, siège de la pensée et de la rationalité, mais au cœur, au "noyau passionnel », à l'âme, animus, ou cor sujet aux passions plus qu'aux raisons. Si la puissance du souverain peut bien commander aux langues, au domaine extérieur de l'échange et de la communication des pensées, il peut aussi susciter un certain nombre de passions, les assujettir au moyen, non pas d'un ordre, d'un discours impératif s'appuyant sur la menace physique, mais d'un usage du langage dont le but n'est pas systématiquement repérable, dont le vulgus ne pense pas à se méfier.

C'est par la duplicité d'un discours intéressé que le souverain parvient à s'immiscer dans l'âme de ses sujets. Ici encore, Spinoza est très proche de Machiavel qui dans Le Prince, s'appuie sur la réalité du mensonge politique pour en tirer des enseignements en matière de gouvernement, recommandant d'utiliser la tromperie, puisque les hommes ne demandent qu'à être trompés, qu'à croire ce qu'on veut bien leur dire. Car les paroles séductrices remplissent un vide, occupent une place que les hommes dédaignent, tout entiers à la poursuite de faux biens, et non guidés par la droite raison. Le discours politique fondé sur la tromperie et le désir de puissance met fin à l'incertitude, au flottement de l'âme et supplée ainsi la réflexion individuelle dont peu d'entre eux sont capables. Il est néanmoins important de ménager au discours séducteur une situation qui n'entre pas en contradiction avec les paroles prononcées explicitement, celui-ci doit en effet être accompagné d'actes qui fonctionnent à la manière de signaux, propres à emporter le consentement des âmes ainsi subjuguées. Machiavel explique que les hommes étant attentifs aux résultats d'une action, à la façon dont celle-ci se donne à voir, le Prince peut alors se contenter de maîtriser les apparences de manière purement extérieure et hypocrite.

Spinoza cite dans le Traité politique (Chapitre VII, §30) l'exemple du roi Aragonais, Don Pedro, qui persuada «à force d'intrigues, de largesses, de promesses et de toute sorte de faveur» le Conseil des Dix-sept, d'abandonner son droit à élire et à déposer le Roi. Or ces promesses, et autres discours flatteurs furent accompagnées non seulement d'actes propres à corrompre les dix-sept sages, mais également d'une action de bravoure, qui impressionna sans doute fortement les âmes : « on dit qu'aussitôt après avoir obtenu ce qu'il demandait, il se coupa la main avec son poignard en présence de la foule, ou du moins, ce que j'ai moins de peine à croire, qu'il se blessa la main en disant qu'il fallait que le sang royal coulât pour que ses sujets eussent le droit d'élire le Roi. » Ainsi le mensonge qui consistait pour Don Pedro, depuis lors surnommé «Poignard », à faire croire au peuple que le fait de retirer au conseil des Dix-sept le droit absolu d'élire, de traduire en 
justice, et de déposer le Roi, était un progrès démocratique, avait besoin d'une telle mise en scène faisant appel non pas à la raison publique, mais au besoin de croire et d'être trompé que possède la foule. La parole, les «machinations perverses» des chefs politiques reposent bien sur l'impact visuel d'actions spectaculaires qui à elles seules donnent aux phrases, aux mots prononcés, leur efficacité. Langage et action sont indissolublement mêlés, et c'est de ce mélange que peut naître la possibilité d'une autorité véritable, c'est-à-dire pleinement effective. Pour commander aux âmes, il est impératif non seulement de dire, mais aussi et surtout de faire. De ce point de vue, le champ politique apparaît comme le lieu d'un théâtre, où seule une parole jouée, mimée, accompagnée de gestes, peut instaurer un ordre, et être sinon comprise, emporter l'adhésion de ses auditeurs/spectateurs auxquels elles est spécifiquement destinée. Cependant, le langage n'est pas instaurateur d'un ordre nouveau, il apparaît principalement dans le Traité politique comme un moyen de maîtriser cette tendance universelle à parler, à exprimer son opinion, fût-elle fausse et dangereuse.

\section{La délibération publique comme expression de la souveraineté}

Le contexte de l'écriture du Traité politique est bien différent de celui du Traité théologico-politique. Entre-temps la Révolution orangiste et son lot de violences ont changé la donne politique et ont montré à Spinoza de manière plus brutale encore le risque qu'il y a à vouloir étouffer la liberté d'expression. Il faut, pour éviter que des idées dangereuses pour l'ordre politique ne circulent et ne constituent une menace leur donner un « droit de cité », une place dans les institutions des différents régimes. Ces moyens institutionnels consistent à traduire légalement la pluralité d'opinions qui animent la multitude.

Il est intéressant de remarquer de ce point de vue que l'acte de parler est défini par Spinoza sur le mode de l'absence de liberté : il n'est pas plus au pouvoir de l'homme de parler que de se taire. C'est avant tout sur lui-même que l'homme est impuissant dans ses discours. On comprend alors pourquoi le régime politique quel qu'il soit doit prendre en compte cette propension à trop en dire, à parler plus que de raison, puisque le langage permet aussi l'expression d'opinions dangereuses pour la stabilité du régime, pour l'ordre public. En effet le chapitre XX du Traité théologico-politique le dit : il n'est pas de plus grande violence que de forcer quelqu'un à se taire, puisque cela va à l'encontre de la nature profonde, de la tendance de tout être humain à penser et surtout à dire ce qu'il pense. Or on voit dans le Traité politique combien la multitude lorsque précisément elle est réduite au silence est dangereuse, et demeure susceptible de transformer le mécontentement qu'elle ne peut pas exprimer, en violence qui fragilise la stabilité de l'État.

De même, l'attitude de Spinoza à l'égard des secrets d'État est très révélatrice. Il critique en effet l'habitude qu'ont les souverains de conserver dans les «arcanes du pouvoir» les raisons de mener telle ou telle guerre (guerre menée et subie par la multitude) : «Ce sont les secrets d'État qui 
font que les sujets succombent sous le fardeau ». Le silence et la nécessité de le garder n'est pas efficace en matière politique et mieux vaut laisser s'exprimer cette tendance à parler naturel à l'être humain comme au corps politique. C'est pourquoi, chaque régime possède dans le Traité politique un dispositif favorisant la concertation, la forme délibérative du discours. Il est même possible de lire le Traité politique et la description des régimes opérée dans les chapitres VI à X à l'aune des notions de délibération, de discussion et de débat. Celui-ci constitue un rouage institutionnel qui laisse place à l'expression des passions, à leur expression orale, contrôlées et améliorées par les exigences de l'efficacité politique.

Ainsi en est-il du Conseil du Roi pour la Monarchie, dont les prérogatives sont énoncées aux $\S 3,4$ et 5 du chapitre VII, et qui est destiné à contrebalancer le pouvoir laissé au monarque, puisque concentré dans les mains d'un seul, celui-ci risque de faire sombrer l'État dans la tyrannie. Il n'est en effet jamais bon qu'un monarque décide seul, sans être entouré et conseillé. Les conseillers sont même obligés, pour la bonne marche du régime, de se concerter de manière régulière et suivie, de soumettre leurs avis au vote et de les suggérer périodiquement au roi, selon un processus rigoureusement et formellement défini. En outre, il est important que les conseillers soient nombreux, afin d'exprimer le plus fidèlement possible les intérêts et les besoins de la multitude dans son ensemble. Les structures de débat sont en effet l'expression de la dissension, du conflit inhérent à toute société politique, et il est important que ces conflits soient dits et entendus par le pouvoir souverain. Ces dispositifs consistent à passer d'un discours comme simple exercice de la puissance sur autrui, dont la modalité principale est l'ordre, vu sous le modèle unilatéral de l'allocution, où aucun échange n'a lieu, à une utilisation du langage où plusieurs interlocuteurs se parlent, s'écoutent, confrontent leurs avis, leurs conceptions du bien commun. C'est ce que montre notamment l'éloge de la délibération qu'effectue Spinoza au chapitre IX, § 14 du Traité politique. Ce chapitre avait pour but de décrire la forme «fédérale» du régime aristocratique, à savoir celle où la souveraineté est détenue par plusieurs villes, et Spinoza défend le dispositif de la délibération, qui permet de faire surgir les conflits qui minent d'autant plus le corps politique qu'ils restent tus, silencieux et pouvant par conséquent créer à terme des séditions, véritable fléau de tous les régimes: «Peu importe que, chaque ville veillant à ses intérêts et jalousant les autres, il y ait assez souvent des discordes entre elles et qu'elles perdent leur temps en discussions. Sans doute, tandis que Rome délibère, Sagonte périt, mais en revanche, lorsqu'un petit nombre décide de tout en fonction de ses seules passions, c'est la liberté qui périt, et le bien commun. Car les esprits humains n'ont pas assez d'acuité pour pouvoir tout pénétrer d'un coup. Mais ils s'aiguisent en délibérant, en écoutant, en discutant ; c'est en examinant toutes les solutions qu'on finit par trouver celles que l'on cherche, sur lesquelles se fait l'unanimité, et auxquelles nul n'avait songé auparavant. » La discussion, les modalités du débat public permettent de neutraliser les passions politiques afin qu'elles ne deviennent pas 
menaçantes pour l'ordre et la sécurité. La préférence de Spinoza pour un État fédéral provient à mon sens de cette nécessité pour les citoyens, ici les patriciens de se réapproprier dans l'espace public de la délibération en assemblée le discours politique, et d'en faire un moyen d'échange plutôt que de domination. C'est donc un éloge de la délibération publique qu'effectue Spinoza : la pratique et la confrontation effectives des discours peut être définie comme un moyen de sauvegarder l'équilibre des forces au sein du corps politique, mais aussi comme une éducation politique. L'importance accordée aux processus délibératifs et la place institutionnelle qui leur est donnée permettent alors de concevoir le langage autrement que comme la traduction policée de purs rapports de force.

Or dans sa prise en compte du langage au sein d'une délibération publique, Spinoza est loin de céder à une sorte d'irénisme pour qui la discussion serait le signe d'une vocation proprement humaine au bien commun et aux préoccupations qu'il suggère. Il faut garder en mémoire les premières pages du Traité politique: il s'agit pour le philosophe de se représenter les hommes «tels qu'ils sont », et les supposés vices de la nature humaine comme des «propriétés qui lui appartiennent au même titre que le chaud, le froid, le mauvais temps, le tonnerre et d'autres phénomènes du même genre appartiennent à la nature de l'atmosphère ». La discussion est nécessaire à la vie politique puisque celle-ci est essentiellement passionnelle: le langage tient ici lieu de rationalité, voire de « raisonnabilité ». Ce passage doit se comprendre comme une réponse, à la fois à Machiavel, qui objecte contre la longueur des délibérations qui retardent la prise de décision politique et à Hobbes qui s'appuie sur cet argument pour critiquer la démocratie. Tout rapprochement hasardeux avec une quelconque «éthique de la discussion » est ici à bannir. Il y a bien chez l'homme une tendance naturelle, instinctive à parler, que signale le Traité théologico-politique, mais cette tendance, au lieu d'élever l'homme à un degré supérieur de rationalité, est au contraire le signe de son incapacité à « tout pénétrer d'un coup », à appréhender de façon immédiate et intuitive un grand nombre d'idées. La délibération (consilia, sur le plan de l'intériorité individuelle, ou deliberatio, du point de vue collectif de la discussion) la considération successive et pour ainsi dire, la mise à plat des arguments au sein d'un débat s'impose au sens où elle seule est adaptée aux capacités intellectuelles des êtres humains pris en tant que corps politique complexe, en tant que multitude. Le langage est ici un outil adapté à la vie politique, et qu'il s'agit de perfectionner en l'institutionnalisant, c'est-à-dire en en faisant l'un des rouages indispensables de la décision en matière politique. Le débat public n'est certes pas le lieu d'un dévoilement de la vérité au sujet du bien commun. Ce qui intéresse ici Spinoza, c'est la façon dont les hommes, ou les citoyens, en discutant, parviennent à confronter leurs conceptions de l'utile, à comparer les différents degrés d'acceptabilité des discours qu'ils entendent.

C'est dans ce cadre que la distinction entre sens et vérité, élaborée par Spinoza afin d'établir une méthode d'interprétation des livres sacrés, prend 
son sens et trouve un aboutissement concret au sein d'une pratique politique. Ne pas être dupe du langage, c'est l'inscrire non pas dans le domaine de la vérité, car alors la plupart des discours seraient faux, ou bien absurdes, ou encore n'auraient rien à nous dire, mais le rendre à l'élément qui est naturellement le sien, c'est-à-dire le sens, et les multiples variations qu'il peut induire, contrairement à la pauvreté de la bivalence vrai/faux qui voudrait être la norme de tout discours. La délibération publique constitue ainsi une sphère où il est essentiel d'écouter d'autres discours, d'autres avis que le sien, voire même de bavarder. C'est en quelque sorte davantage l'acte même de délibérer qui importe, plus que la teneur de vérité à laquelle ces délibérations peuvent parvenir. Cette activité est essentielle au champ politique comme un mécanisme qui permet aux hommes de devenir " civiles », c'est-à-dire de laisser leur intérêt se réfléchir à celui des autres, et d'acquérir un sens de la légalité, qui se trouve chaque fois nouvellement investi par et dans ce processus de délibération.

Ce processus repose bien sur la nécessité pragmatique de faire vivre ensemble des individus aux intérêts antagonistes, vivant selon l'ordre passionnel, prompts à s'arroger l'exclusivité d'un intérêt en le fondant sur une révélation ou un texte sacré. La délibération permet quant à elle de séculariser l'expression et l'appréciation des diverses considérations sur l'utile. Il y a bien, du même coup, une réappropriation du discours politique, qui contribue paradoxalement à asseoir l'autorité en la diffusant, en la disséminant au sein d'un sphère publique où se dessine par réflexion ce que l'on peut appeler une « opinion publique », tissée progressivement au fil des échanges verbaux. Cette structure de délibération semble avoir remplacé celle du contrat dans le Traité politique, celui-ci ne pouvant s'appuyer durablement sur un engagement verbal unilatéral et ponctuel. Au sein même de l'aristocratie, Spinoza défend la pluralité des assemblées, chargées de se contrôler les unes les autres : en dehors de l'assemblée suprême, le Sénat, les syndics sont nécessaires afin de maintenir le pluralisme et d'éviter le plus possible l'existence d'une seule instance de l'autorité. Il ne s'agit cependant pas d'un partage du pouvoir, puisque ces dispositifs sont avant tout de type consultatif et consistent à faire entendre une autre parole que celle du pouvoir. En effet, comme on l'a vu, cette parole s'exprimera de toute façon, compte tenu de cette pulsion de discours qui pousse les hommes à extérioriser leurs pensées (le conatus a bien ici sa conséquence sur le plan de l'usage du langage). Mais parler prend du temps, c'est pourquoi la délibération doit être contenue et soumise à l'efficacité politique (VIII, § 36). Les structures institutionnelles permettent ainsi la canalisation de la pulsion de discours : à chaque fois, Spinoza entend éviter l'unilatéralité par des moyens, même modestes, de contre-pouvoirs fondés sur le pluralisme de la parole. Dans chaque régime, il s'agit de redistribuer le droit de parole afin de contrebalancer la puissance excessive, qui se traduit toujours par un monopole du discours. Le pluralisme politique se double chaque fois de dispositifs qui font intervenir plusieurs instances dans le débat public et assument le droit de parole. Le principe semble être le suivant: pour que 
l'exercice de la puissance du souverain au sein du corps politique soit équilibré, il faut qu'un grand nombre de citoyens puissent faire entendre leurs voix et surtout entendre celle des autres. Ce droit de suffrage et de participation de tous les sujets de droit aux affaires de l'État qui définissent au dernier chapitre du Traité politique le régime démocratique doit nécessairement s'appuyer sur cette maîtrise de la parole politique, condition d'un partage effectif de la souveraineté. Le pouvoir du langage se définirait alors comme l'expression apaisée de la puissance de la multitude au sein d'institutions où la force se convertit en mots.

\section{Bibliographie}

\section{Cuvres de Spinoza}

Spinoza Opera, édité par C. Gebhardt, Heidelberg, 1925

\section{Traductions utilisées}

Traité théologico-politique, traduit et annoté par J. Lagrée et P.-F. Moreau, PUF, 1999

Traité politique, traduit et annoté par Ch. Ramond, PUF, 2005

Traité politique, traduit par É. Saisset, préfacé et annoté par Laurent Bove, Livre de Poche, 2002

Pensées métaphysiques, traduction Ch. Appuhn, G.-F., 1964

Traité de la réforme de l'entendement, traduction B. Pautrat, Allia, 1999

Traité de la réforme de l'entendement, traduction B. Rousset, Vrin, 1992

\section{Autres ouvrages utilisés}

Ducrot O. et Schaeffer J.-M., Nouveau dictionnaire des sciences du langage, Paris, Seuil, 1995.

Hobbes, Léviathan, traduction, introduction, notes et notices par G. Mairet, Paris, Gallimard, 2000.

Machiavel, Euvres, Paris, Gallimard, collection «La Pléiade », 1980

Moreau P.-F., « Politiques du langage », Revue philosophique de la France et de l'étranger, 1985.

Negri A., L'Anomalie sauvage. Puissance et pouvoir chez Spinoza, Paris, PUF, 1982.

Nietzsche, Le Livre du philosophe, traduction Angèle Kremer-Marietti, Paris, G.-F., 1991.

Tosel A., «La théorie de la pratique et la fonction de l'opinion publique dans la philosophie de Spinoza », Studia Spinozana, Paris, Kimé, 1994. 